\title{
Covid-19 em Crianças com Câncer
}

https://doi.org/10.32635/2176-9745.RBC.2020v66nTemaAtual.1227

COVID-19 in Children with Cancer

Covid-19 en Niños con Cáncer

Luciana Chain Veronez'; Luís Carlos Lopes-Júnior²

\section{INTRODUÇÃO}

Após dezembro de 2019, quando registrados os primeiros casos da então doença causada por um coronavírus recém-identificado (agora já nomeado como Sars-CoV-2, do inglês, severe acute respiratory syndrome coronavirus 2) em Wuhan, China ${ }^{1}$, a doença causada pelo novo coronavírus (coronavirus disease 2019 - Covid-19) emergiu oficialmente como uma pandemia declarada pela Organização Mundial da Saúde (OMS) em 11 de março de $2020^{2,3}$, infectando mais de 118.319 pessoas e com 4.292 mortes naquela ocasião ${ }^{4}$. Atualmente, os casos confirmados de infecção pelo Sars-CoV-2 em 213 países e territórios em todo o mundo já somam mais de 24.587.513, com 833.556 mortes registradas em 29 de agosto de 2020 pela OMS5 5

A Covid-19 caracteriza-se como uma infecção aguda do trato respiratório com quadros clínicos que variam desde formas leves e/ou assintomáticas até formas graves. Embora sua taxa de letalidade seja baixa, cerca de $3,7 \%$ dos casos, alguns fatores estão associados com um risco aumentado para uma evoluçáo mais grave da doença e morte, tais como idade avançada e presença de comorbidades, como é o caso de pacientes com câncer ${ }^{6}$.

A prevalência de câncer em pacientes com Covid-19 é de, aproximadamente, $2 \%$ (IC95\% 2,0\%-3,0\%) Pesquisas recentes sugerem que pacientes oncológicos apresentam um risco aumentado para a infecção pelo Sars-CoV-2 e complicaçōes graves da Covid-19 do que a população em geral em razão do seu estado comumente imunossuprimido decorrente do próprio câncer ou dos tratamentos anticâncer, além da maior necessidade de idas a ambientes hospitalares com maior probabilidade de exposiçáo ao vírus ${ }^{8-10}$. No entanto, dados sobre o risco de infecção, prognóstico e impacto da Covid-19 na população pediátrica, especialmente em crianças com câncer, ainda são escassos.

\section{DESENVOLVIMENTO}

Em recente revisão sistemática ${ }^{11}$, que reuniu dados de 131 estudos publicados até 14 de maio de 2020, totalizando 7.780 pacientes pediátricos de 26 países diferentes, os autores reforçaram evidências prévias ${ }^{12-14} \mathrm{de}$ que crianças diagnosticadas com Covid-19 apresentam, em geral, um bom prognóstico e baixa taxa de mortalidade ${ }^{11}$. Entre os casos analisados nessa revisão, foram reportadas apenas 11 crianças $(0,14 \%)$ que se enquadraram no quadro de síndrome inflamatória multissistêmica e sete mortes $(0,09 \%)$, sendo que $76,6 \%$ dos pacientes foram expostos a familiares diagnosticados com Covid-19. Como observado em estudos anteriores, as manifestações clínicas em crianças com Covid-19 foram geralmente brandas, sendo as mais frequentes febre e tosse, havendo muitos casos assintomáticos ${ }^{11,12,15,16}$.

Segundo dados publicados na JAMA Oncology ${ }^{17}$, crianças com câncer parecem não ser mais vulneráveis à infecção ou a morbidades decorrentes do Sars-CoV-2 do que as demais crianças. Nesse estudo, foram avaliados 178 pacientes assistidos pelo Memorial Sloan Kettering Cancer Center de Nova York, mostrando que apenas 5\% dos pacientes oncológicos precisaram ser hospitalizados, e 2,5\% das crianças assintomáticas sem exposição conhecida ao vírus (120) testaram positivo para Covid-19. Similarmente à diferença observada em adultos, os pesquisadores relataram que, dos 20 pacientes com a infeção, três eram do gênero masculino e 17 feminino ${ }^{17}$. No entanto, cabe ressaltar o número limitado de crianças avaliadas, o que reforça a necessidade de novos estudos para confirmar tais achados.

Todavia, em crianças com câncer, a preocupação corrente náo se limita apenas à possibilidade de risco aumentado de infecção pelo Sars-CoV-2 ou à maior gravidade do desfecho clínico, mas também ao impacto da pandemia de Covid-19 no manejo e cuidado desses

'Bióloga. Doutora em Imunologia pela Universidade de São Paulo (USP). Pós-Doutoranda do Departamento de Puericultura e Pediatria da Faculdade de Medicina de Ribeirão Preto (FMRP) da USP. Ribeirão Preto (SP), Brasil. Orcid iD: https://orcid.org/0000-0001-8926-2186

${ }^{2}$ Enfermeiro Oncologista. Doutor em Ciências pela USP. Professor-Adjunto do Centro de Ciências da Saúde da Universidade Federal do Espírito Santo (Ufes). Vitória (ES), Brasil. Orcid iD: https://orcid.org/0000-0002-2424-6510

Endereço para correspondência: Luís Carlos Lopes-Júnior. Centro de Ciências da Saúde da Ufes. Avenida Marechal Campos, 1468 - Maruípe. Vitória (ES), Brasil. CEP 29047-105. E-mail: lopesjr.lc@gmail.com 
pacientes, principalmente no que concerne ao acesso a seus tratamentos correntes ${ }^{18-20}$. Em artigo publicado no início de maio de 2020 no The Lancet, ainda na fase inicial da pandemia, pesquisadores já reportaram consequências negativas substanciais no cuidado de crianças com câncer na América Latina ${ }^{21}$. Entre os principais problemas relatados, destacaram-se atrasos em consultas e/ou modificações em esquemas de tratamentos, e diminuição nas equipes de onco-hematologia pediátrica ${ }^{21}$.

Nesse cenário incerto e sem precedentes, imposto pela atual pandemia da Covid-19, grupos de pesquisa e profissionais de saúde têm se preocupado em reunir e fornecer recomendaçóes sobre adaptaçôes razoáveis e seguras para serem aplicadas nos serviços de oncologia, protegendo não só o paciente, como também suas famílias e a equipe de saúde interdisciplinar no cuidado ao câncer ${ }^{18,19,22,23}$. Um exemplo disso é o guia clínico internacional publicado como relato especial na revista Pediatric Blood \& Cancer $^{18}$, que reuniu informaçóes das principais organizaçôes de câncer infantojuvenil, tais como a International Society for Pediatric Oncology (SIOP), o Children's Oncology Group (COG) e o St. Jude Global, o qual forneceu orientaçóes de adaptaçôes que poderão ajudar a gerenciar as demandas da pandemia e nortear preparaçóes para os impactos a longo prazo, como diagnósticos $\operatorname{tardios}^{18}$.

Em parceria com a SIOP, o St. Jude Children's Research Hospital desenvolveu uma plataforma de registro global de dados de crianças com câncer infectadas pelo Sars-CoV-2 com o objetivo de coletar dados para futuras análises, compartilhar experiências clínicas e desenvolver seminários e workshops sobre a problemática. Acessada em 29 de agosto de 2020, essa plataforma registrava 803 casos positivos espalhados por 35 países diferentes, dos quais cerca de $30 \%$ eram de crianças entre 5 e 9 anos de idade e a grande maioria assintomática $(42,5 \%)^{24}$.

\section{CONCLUSÃO}

Ainda são inúmeros e complexos os desafios que a pandemia do novo coronavírus imprime na área da Oncologia Pediátrica, sendo eles não só relacionados às mudanças existenciais que permeiam o mundo da criança e da família dos pacientes pediátricos oncológicos, como também daqueles que profissionalmente os assistem em busca de um cuidado personalizado e seguro.

\section{CONTRIBUIÇÕES}

Os autores contribuíram substancialmente e igualmente em todas as etapas do artigo e aprovaram a versão final a ser publicada.

\section{DECLARAÇÃO DE CONFLITO DE INTERESSES}

Nada a declarar.

\section{FONTES DE FINANCIAMENTO}

Não há.

\section{REFERÊNCIAS}

1. Fauci AS, Lane HC, Redfield RR. Covid-19 - Navigating the uncharted. N Engl J Med. 2020;382(13):1268-9. doi: https://doi.org/10.1056/NEJMe2002387

2. Mahase E. Covid-19: WHO declares pandemic because of "alarming levels" of spread, severity, and inaction. BMJ. 2020;368:m1036. doi: https://doi.org/10.1136/ bmj.m1036.

3. Lopes-Júnior LC, Bomfim E, Silveira DSC, et al. Effectiveness of mass testing for control of COVID-19: a systematic review protocol. BMJ Open. 2020;10(8):e040413. doi: https://doi.org/10.1136/ bmjopen-2020-040413

4. World Health Organization. Coronavirus disease 2019 (COVID-19): situation report -51 [Internet]. [Geneva]: WHO; 2020 Mar 11 [cited 2020 Aug 29]. Available from: https://www.who.int/docs/default-source/ coronaviruse/situation-reports/20200311-sitrep-51covid-19.pdf?sfvrsn=1ba62e57_10.

5. World Health Organization. Coronavirus disease (COVID-19) pandemic. Numbers at a glance. Geneva: WHO; c2020 [Last update 2020 Aug 27;cited 2020 Aug 29]. Available from: https://www.who.int/emergencies/ diseases/novel-coronavirus-2019.

6. Oh WK. COVID-19 infection in cancer patients: early observations and unanswered questions. Ann Oncol. 2020;31(7):838-9. doi: https://doi.org/10.1016/j. annonc.2020.03.297

7. Desai A, Sachdeva S, Parekh T, et al. COVID-19 and cancer: lessons from a pooled meta-analysis. JCO Glob Oncol. 2020;6:557-9. doi: https://doi.org/10.1200/ GO.20.00097

8. Liang W, Guan W, Chen R, et al. Cancer patients in SARS-CoV-2 infection: a nationwide analysis in China. Lancet Oncol. 2020;21(3):335-7. doi: https://doi. org/10.1016/S1470-2045(20)30096-6

9. Sica A, Massarotti M. Myeloid suppressor cells in cancer and autoimmunity. J Autoimmun. 2017;85:117-25. doi: https://doi.org/10.1016/j.jaut.2017.07.010

10. Moujaess E, Kourie HR, Ghosn M. Cancer patients and research during COVID-19 pandemic: a systematic review of current evidence. Crit Rev Oncol Hematol. 2020;150:102972. doi: https://doi.org/10.1016/j. critrevonc.2020.102972 
11. Hoang A, Chorath K, Moreira A, et al. COVID-19 in 7780 pediatric patients: a systematic review. EClinical Medicine. 2020;24:100433. doi: https://doi. org/10.1016/j.eclinm.2020.100433

12. Lu X, Zhang L, Du H, et al. SARS-CoV-2 infection in children. N Engl J Med. 2020;382(17):1663-5. doi: https://doi.org/10.1056/NEJMc2005073

13. Ludvigsson JF. Systematic review of COVID-19 in children shows milder cases and a better prognosis than adults. Acta Paediatr. 2020;109(6):1088-95. doi: https:// doi.org/10.1111/apa.15270

14. de Rojas T, Pérez-Martínez A, Cela E, et al. COVID-19 infection in children and adolescents with cancer in Madrid. Pediatr Blood Cancer. 2020;67(7):e28397. doi: https://doi.org/10.1002/pbc.28397

15. de Souza TH, Nadal JA, Nogueira RJN, et al. Clinical manifestations of children with COVID-19: a systematic review. Pediatr Pulmonol. 2020;55(8):1892-9. doi: https://doi.org/10.1002/ppul.24885

16. Mustafa NM, A Selim L. Characterisation of COVID-19 pandemic in paediatric age group: a systematic review and meta-analysis. J Clin Virol. 2020;128:104395. doi: https://doi.org/10.1016/j.jcv.2020.104395

17. Boulad F, Kamboj M, Bouvier N, et al. COVID-19 in children with cancer in New York city. JAMA Oncol. 2020;e202028. doi: https://doi.org/10.1001/ jamaoncol.2020.2028

18. Sullivan M, Bouffet E, Rodriguez-Galindo C, et al. The COVID-19 pandemic: a rapid global response for children with cancer from SIOP, COG, SIOP-E, SIOP-PODC, IPSO, PROS, CCI, and St Jude Global. Pediatr Blood Cancer. 2020;67(7):e28409. doi: https:// doi.org/10.1002/pbc.28409

19. Bouffet E, Challinor J, Sullivan M, et al. Early advice on managing children with cancer during the COVID-19 pandemic and a call for sharing experiences. Pediatr Blood Cancer. 2020;67(7):e28327. doi: https://doi. org/10.1002/pbc.28327

20. Lopes-Júnior LC, Lima RAG. Cuidado ao câncer e a prática interdisciplinar. Cad Saúde Pública. 2019;35(1):e00193218. doi: https://doi. org/10.1590/0102-311x00193218

21. Vasquez L, Sampor C, Villanueva G, et al. Early impact of the COVID-19 pandemic on paediatric cancer care in Latin America. Lancet Oncol. 2020;21(6):753-5. doi: https://doi.org/10.1016/S1470-2045(20)30280-1

22. Baruchel A, Bertrand Y, Boissel N, et al. COVID-19 and acute lymphoblastic leukemias of children and adolescents: first recommendations of the Leukemia committee of the French Society for the fight against Cancers and Leukemias in children and adolescents (SFCE). Bull Cancer. 2020;107(6):629-32. doi: https:// doi.org/10.1016/j.bulcan.2020.04.003
23. Silva Junior FJG, Sales JCS, Monteiro CFS, et al. Impact of COVID-19 pandemic on mental health of young people and adults: a systematic review protocol of observational studies. BMJ Open. 2020;10(7):e039426. doi: https://doi.org/10.1136/bmjopen-2020-039426

24. Covid-19 in Pediatric Cancer Global Registry Data [Internet]. Memphis, TN: St. Jude Children's Research Hospital; International Society of Paediatric Oncology (SIOP). [2020] - [last update $2020 \mathrm{Mar}$ 9;cited 2020 Aug 29]. Available from: https://app. powerbi.com/w?r=eyJrIjoiNGQ3NDAwZDItYjRjN i00MjNhLWE2NTMtNmFjNmU1YTgzZDMwliwi dCI6IjIyMzQwZmE4LTkyMjYtNDg3MS1iNjc3LW QzYjNIMzc3YWY3MiIsImMiOjN9 